\title{
SPIRAL ASYMPTOTIC VALUES OF FUNCTIONS MEROMORPHIC IN THE UNIT DISK
}

\author{
J.L. Stebbins
}

\section{Introduction}

This paper contains part of the author's Ph.D. dissertation directed by Frederick Bagemihl at Wayne State University. The research was supported by a grant from the Michigan Institute of Science and Technology.

Alice Roth has made an extensive study of entire meromorphic functions with prescribed behavior along half rays emanating from the origin (6). The question arose whether analogous results could be found for functions meromorphic in the unit disk with the same behavior prescribed along an exhaustive class of spirals emanating from the origin. In this paper, I present a class of spirals which satisfactorily fills this role. However, I make no claim to the effect that only this class will suffice.

\section{The main results}

The circle with origin as center and radius $k$ in the complex plane will be denoted by $C(k)$ throughout this paper. Similarly, the open disk with origin as center and radius $k$ will be denoted by $D(k)$.

(2.1) Definition. For each value $\theta, 0 \leq \theta<2 \pi$, define $S_{\theta}=\{z=$ $\left.r \exp \left[i\left(\theta+\tan \frac{\pi r}{2}\right)\right], 0 \leq r<1\right\}$. Further, define $S_{-\theta}=S_{2 \pi-\theta}$ for each $\theta, 0 \leq \theta$ $\leq 2 \pi$. These spirals will be called Study spirals (7, p. 45).

Notice that each Study spiral originates at the origin and its argument tends monotonically to $+\infty$ as $r$ tends to 1 . Also if $\theta_{1} \neq \theta_{2}$, then $S_{\theta_{1}}$ and $S_{\theta_{2}}$ have only the origin in common.

(2. 2) Definition. Let $\mathscr{S}$ represent the class of functions meromorphic and non-constant in $D(1)$ and which tend to a definite limit, finite or infinite, on each Study spiral as $r$ tends to 1. (Theorem 2.15 asserts that this class is rather large.) For each function $F(z)$ define the spiral limit value function $f(\theta)$, $0 \leq \theta<2 \pi$, associated with $F(z)$ as follows:

Received June 13, 1966. 


$$
f(\theta)=\lim _{r \rightarrow 1} F\left(r \exp \left[i\left(\theta+\tan \frac{\pi r}{2}\right)\right]\right) .
$$

(2.3) Definition. For $-2 \pi \leq \theta_{1}<\theta_{2} \leq 2 \pi$ and $\theta_{2}-\theta_{1} \leq 2 \pi$ I shall call the region bounded by $S_{\theta_{1}}, S_{\theta_{2}}$ and $C(1)$ and containing $S_{\theta_{3}}$ where $\theta_{3}=\theta_{1}+\frac{\theta_{2}-\theta_{1}}{2}$ a spiral wedge and I shall denote this region by $W\left(\theta_{1}, \theta_{2}\right)$. Let $\bar{W}\left(\theta_{1}, \theta_{2}\right)$ denote the union of $W\left(\theta_{1}, \theta_{2}\right), S_{\theta_{1}}$ and $S_{\theta_{2}}$ and be called a closed spiral wedge.

(2. 4) Lemma 1. Let $F(z) \in \mathscr{S}$. In each closed region $\bar{W}\left(\alpha_{0}, \beta_{0}\right)$ there is a closed region $\bar{W}(\alpha, \beta)$, where $\alpha_{0}<\alpha<\beta<\beta_{0}$, such that either $|F(z)| \leq 2$ or $|F(z)| \geq 1$ for all sufficiently large $|z|$, where $z \in \bar{W}(\alpha, \beta)$.

Proof. Assume the conclusion does not hold. Then there exists $1>\left|z_{1}\right|$ $=r_{1}>1 / 2$ such that $z_{1} \in W\left(\alpha_{0}, \beta_{0}\right)$ and $\left|F\left(z_{1}\right)\right|<1$. Since $F(z)$ is continuous at $z_{1}$, there is an entire arc on $|z|=r_{1}$ such that $|F(z)|<1$ for all $z$ on this arc. Hence there are values $\alpha_{1}$ and $\beta_{1}$ such that $\alpha_{0}<\alpha_{1}<\beta_{1}<\beta_{0}$ and for $z \in W\left(\alpha_{1}, \beta_{1}\right)$ and $|z|=r_{1},|F(z)|<1$.

Also there exists $1>\left|z_{2}\right|=r_{2}>3 / 4$ such that $z_{2} \in W\left(\alpha_{1}, \beta_{1}\right)$ and $\left|F\left(z_{2}\right)\right|>2$. Since $1 / F(z)$ is continuous at $z_{2}$, there is an entire arc of $|z|=r_{2}$ on which $|F(z)|>2$. So there are values $\alpha_{2}, \beta_{2}$ such that $\alpha_{1}<\alpha_{2}<\beta_{2}<\beta_{1}$ and for all $z$ where $z=r_{2} \exp \left[i\left(\theta+\tan \frac{\pi r_{2}}{2}\right)\right]$ and $\alpha_{2}<\theta<\beta_{2},|F(z)|>2$.

Continuing on in this way for each $n=1,2, \ldots \ldots$ the argument reads as follows :

For $|z|>2 n / 2 n+2$, there exists $z_{2 n-1}=r_{2 n-1} \exp \left[i\left(\theta_{2 n-1}+\tan \frac{\pi r_{2 n-1}}{2}\right)\right]$ where $\alpha_{2 n-2}<\theta_{2 n-1}<\beta_{2 n-2}$ and $1>r_{2 n-1}>\frac{2 n}{2 n+2}$ such that $\left|F\left(z_{2 n-1}\right)\right|<1$. Because $F(z)$ is continuous at $z_{2 n-1}$, there exists an entire subarc of $|z|=r_{2 n-1}$ containing the points $z=r_{2 n-1} \exp \left[i\left(\theta+\tan \frac{\pi r_{2 n-1}}{2}\right)\right]$ with $\alpha_{2 n-1}<\theta<\beta_{2 n-1}$ where $\alpha_{2 n-2}$ $<\alpha_{2 n-1}<\beta_{2 n-1}<\beta_{2 n-2}$ on which $|F(z)|<1$.

Also there exists $z_{2 n}=r_{2 n} \exp \left[i\left(\theta_{2 n}+\tan \frac{\pi r_{2 n}}{2}\right)\right]$ where $1>r_{2 n}>\frac{2 n+1}{2 n+2}$ and $\alpha_{2 n-1}<\theta_{2 n}<\beta_{2 n-1}$ and for which $\left|F\left(z_{2 n}\right)\right|>2$. Since $1 / F(z)$ is continuous at $z_{2 n}$, there is an entire subarc of $|z|=r_{2 n}$ containing the points $z=r_{2 n} \exp [i(\theta+$ $\left.\left.\tan \frac{\pi r_{2 n}}{2}\right)\right]$ with $\alpha_{2 n}<\theta<\beta_{2 n}$ where $\alpha_{2 n-1}<\alpha_{2 n}<\beta_{2 n}<\beta_{2 n-1}$ on which $|F(z)|$ $>2$. 
So I have defined the following sequence of open, non-empty, nested intervals $\left(\alpha_{1}, \beta_{1}\right), \quad\left(\alpha_{2}, \beta_{2}\right), \ldots . .$. where $\alpha_{k+1}, \beta_{k+1} \in\left(\alpha_{k}, \beta_{k}\right) k=0,1,2, \ldots \ldots$ Therefore $\bigcap_{n=1}^{\infty}\left(\alpha_{n}, \beta_{n}\right)$ is not empty. Let $\gamma$ be in this set. Then for this $\gamma$ and for all $n$, I have both $\left|F\left(r_{2 n-1} \exp \left[i\left(\gamma+\tan \frac{\pi r_{2 n-1}}{2}\right)\right]\right)\right|<1$ and $\left|F\left(r_{2 n} \exp \left[i\left(\gamma+\tan \frac{\pi r_{2 n}}{2}\right)\right]\right)\right|>2$. Since $\lim _{n \rightarrow \infty} r_{2 n-1}=\lim _{n \rightarrow \infty} r_{2 n}=1$, this implies that no limit exists for $F(z)$ as $|z|$ tends to 1 along $S_{\gamma}$, contrary to my assumption. So the proposition must be true.

(2. 5) Definition. A spiral wedge $W(\alpha, \beta)$ is called a wedge of convergence for $F(z) \in \mathscr{S}$ if either a finite constant $c$ exists so that $\lim _{r \rightarrow 1}\{F(r \exp [i(\theta+$ $\left.\left.\left.\left.\tan \frac{\pi r}{2}\right)\right]\right)-c\right\}=0$ uniformly for $\alpha \leq \theta \leq \beta$ or if $\lim _{r \rightarrow 1} \frac{1}{F\left(r \exp \left[i\left(\theta+\tan \frac{\pi r}{2}\right)\right]\right)}=0$ uniformly for $\alpha \leq \theta \leq \beta$.

The range of $f(z)$, denoted by $R(f)$, is the set of those values assumed by $f(z)$ at points in $D(1)$ arbitrarily near $C(1)$.

(2. 6) Lemma 2. Let $F(z) \in \mathscr{S}$ and define $F_{w}(z)$ to be $F(z)$ restricted to the closed spiral wedge $\bar{W}(\alpha, \beta)$. If $\mathscr{C} R\left(F_{w}\right)$ (i.e., the complement of the range of $F$ restricted to this same wedge) contains more than two elements, then $W(\alpha, \beta)$ is a wedge of convergence for $F(z)$.

Proof. The region $W(\alpha, \beta)$ is a simply connected region which possesses $C(1)$ as a single prime end. Using Carathéodory's classical theorem on prime ends (3), there is a conformal mapping from $W(\alpha, \beta)$ onto $D^{\prime}(1)=|\zeta|<1$ such that the points of $S_{\alpha}$ and $S_{\beta}$ are mapped conformally onto $C^{\prime}(1)=|\zeta|=1$ with the exception of $\zeta=1$. The prime end $C(1)$ corresponds to the point $\zeta=1$. Let $\zeta=\zeta_{(z)}$ represent this mapping and let $z=z(\zeta)$ be its inverse.

Consider the composition $w=F^{*}(\zeta)=F(z(\zeta))$ defined for all $|\zeta| \leq 1$ with the exception of $\zeta=1$. $\mathscr{C} R\left(F^{*}(\zeta)\right)$ contains more than two elements by hypothesis. That $F^{*}(\zeta)$ represents a normal function in $D^{\prime}(1)$ follows from a corollary in Noshiro's book (5, p. 89) which states that any meromorphic function defined in $D^{\prime}(1)$ omitting three values is normal. (If $F(z)$ does not omit three values in $W(\alpha, \beta)$, it does for all $|z|>R$ for some $R<1$ and $z \in W(\alpha, \beta)$.) Then map this region onto $D^{\prime}(1)$ in the same way.)

I now refer the reader to a result of O. Lehto and K.I. Virtanen (4, p. 53, Theorem 2) which states that a normal meromorphic function in a Jordan 
region $G$ having an asymptotic value $\alpha$ at a boundary point $P$ along a Jordan curve lying in the closure of $G$ possesses an angular limit $\alpha$ at the point $P$. I note here that the proof of this theorem yields the result that if the asymptotic path $\Gamma$ lies on the boundary of $G$, the function not only possesses the limit $\alpha$ in every angle $A$, but it also tends uniformly to $\alpha$ in the part of $G$ lying between $A$ and the path $\Gamma$.

Let $\Gamma_{\beta}, \Gamma_{\alpha}$ be the images of $S_{\beta}, S_{\alpha}$ respectively on $C^{\prime}(1)$. Then assuming $F(z)$ has the asymptotic values $\alpha^{\prime}$ and $\beta^{\prime}$ along the spirals $S_{\alpha}$ and $S_{\beta}$ respectively $F^{*}(\zeta)$ has the asymptotic values $\alpha^{\prime}$ and $\beta^{\prime}$ along the paths $\Gamma_{\alpha}$ and $\Gamma_{\beta}$ respectively. Then by the above result of Lehto and Virtanan, $F^{*}(\zeta)$ has both the angular limit $\alpha^{\prime}$ and $\beta^{\prime}$ at $\zeta=1$. Thus $\alpha^{\prime}$ must equal $\beta^{\prime}$ and by the note I made above $F^{*}(\zeta)$ must tend uniformly to $\alpha^{\prime}$ as $\zeta$ tends to 1 in all of $\overline{D^{\prime}(1)}-\{1\}$.

Hence for $\varepsilon>0$ there exists a $\delta>0$ such that for all $\zeta$ satisfying $0<|\zeta-1|<\delta$ and $\zeta \in \overline{D^{\prime}(1)},\left|F^{*}(\zeta)-\alpha^{\prime}\right|<\varepsilon$. Consider $|\zeta-1|=\delta$. This circle cuts $\Gamma_{\alpha}$ and $\Gamma_{\beta}$ and the part of this circle in $D^{\prime}(1)$ together with the initial parts of $\Gamma_{\alpha}$ and $\Gamma_{\beta}$, up to the points of intersection, forms a closed Jordan curve in $\overline{D^{\prime}(1)}$. The image of this closed Jordan curve under $z=z(\zeta)$ is a closed Jordan curve in $D(1)$. This image has a positive distance $d$ from $C(1)$. Then for $z \in \bar{W}(\alpha, \beta)$ and $|z|>1-d, \quad\left|F(z)-\alpha^{\prime}\right|=\left|F(z(\zeta))-\alpha^{\prime}\right| \quad($ where $0<|\zeta-1|<\delta) \quad=\left|F^{*}(\zeta)-\alpha^{\prime}\right|<\varepsilon$. Therefore $W(\alpha, \beta)$ is a wedge of convergence for $F(z)$ as was to be proved.

(2. 7) Definition. For $f(z) \in \mathscr{S}$, if there exists a $\theta$ such that for every $\varepsilon>0 F(z)$ assumes all values (including $\infty$ ) with the possible exception of at most two infinitely often in $W(\theta-\varepsilon, \theta+\varepsilon), S_{\theta}$ will be called a Julia spiral.

(2. 8) Definition. Let $D(F)$ represent the set of those points $1 / 2 \exp [i(\theta+\tan \pi / 4)]$ on $C(1 / 2)$ which are cut by Julia spirals of the function $F(z) \in \mathscr{S}$.

(2. 9) Theorem. If $F(z) \in \mathscr{S}$, then $D(F)$ is a nowhere dense closed set. Let the function $g(\theta)$ associate the spiral limit value $f(\theta)$ with each point $1 / 2 \exp [i(\theta+\tan \pi / 4)]$ of $C(1 / 2)$. Then this correspondence is a function of Baire class 0 or 1 , whose intervals of constancy lie dense on $C(1 / 2)$; this function is constant at least on each arc of $C(1 / 2)$ in $\mathscr{C} D(F)$. In every closed spiral wedge which contains no point of the set $D(F), F(z)$ tends uniformly towards the corresponding constant spiral limit value.

Proof. In each closed disk $\overline{D(k)}$ where $0<k<1, F(z)$ can have at most finitely many poles. If this were not true it would have a limit point of poles in $D(1)$, and so not be meromorphic there. So there is an increasing sequence 
of positive numbers $\left\{r_{n}\right\}$ with $\lim _{n \rightarrow \infty} r_{n}=1$ such that, for every $n, F(z)$ has no poles on $|z|=r_{n}$. Thus $f(\theta)$ is the limit function of the sequence of continuous functions $f_{n}(\theta)=F\left(r_{n} \exp \left[i\left(\theta+\tan \frac{\pi r_{n}}{2}\right)\right]\right) 0 \leq \theta<2 \pi$.

I claim next that every spiral wedge contains a spiral wedge of convergence. Lemma 1 (2. 4) allows me to conclude that each spiral wedge contains a closed spiral wedge $\bar{W}(\alpha, \beta)$ in which either $|F(z)| \leq 2$ or $1 /|F(z)| \leq 1$ for sufficiently large values of $|z|$ (i.e., $R<|z|<1$ ). For $z \in \bar{W}(\alpha, \beta)$ and $|z| \leq R, F(z)$ cannot assume any value more than a finite number of times, since $F(z)$ is not constant. For $z \in \bar{W}(\alpha, \beta)$ and $R<|z|<1|F(z)| \leq 2$ (or $1 /|F(z)| \leq 1$ ), and so the complement of the range of $F(z)$ (or the complement of the range of $1 / F(z)$ ) restricted to $\bar{W}(\alpha, \beta)$ contains many more than two elements. Therefore, by Lemma 2 (2.6), $W(\alpha, \beta)$ is a wedge of convergence for $F(z)$ (or $1 / F(z)$ ).

Thus it follows that wedges of convergence are everywhere dense in $D(1)$ and the intersection of the union of all wedges of convergence with $C(1 / 2)$ is a set everywhere dense on $C(1 / 2)$. The function $g$ is constant on the intersection of each wedge of convergence with $C(1 / 2)$ and so the intervals of constancy of $g$ are dense on $C(1 / 2)$.

Lemma 2 also allows me to conclude that if $S_{\theta}$ is contained in no wedge of convergence of $F(z)$, then $F(z)$ assumes every value (including $\infty$ ) with at most two exceptions infinitely often in $W(\theta-\varepsilon, \theta+\varepsilon)$ for each $\varepsilon>0$. Hence $S_{\theta}$ is a Julia spiral.

Conversely, no Julia spiral is contained in a wedge of convergence. If this were not true and $W$ was a wedge of convergence containing a Julia spiral, $W$ would have to contain an infinite number of $a$-points $\left(z_{0}\right.$ is an $a$-point of $F(z)$ if $F\left(z_{0}\right)=a$ ) for all complex numbers $a$ with at most two exceptions. Since $W$ is a wedge of convergence there exists a constant $\alpha$ such that for every $\varepsilon>0$ there is a $0<R(\varepsilon)<1$ such that if $z \in \bar{W}$ and $|z|>R(\varepsilon), \quad|F(z)-\alpha|<\varepsilon$. Let $a$ be a complex number which is not one of the two exceptional points of $F(z)$ and $|a|>|\alpha|+\varepsilon$. Then $F(z)$ must have an infinite number of $a$-points in $\bar{W} \cap \overline{D(R(\varepsilon)})$ and this implies $F(z) \equiv a$. Since this contradicts the fact that $F(z) \in \mathscr{S}$, I may conclude that no Julia spiral is contained in a wedge of convergence. So the set of Julia spirals is identical with the set of Study spirals that are contained in no wedge of convergence.

The set $D(F)$ is closed since it must contain all its limit points. Also, since $D(F)$ is the complement of an everywhere dense set, it is nowhere dense on 
$C(1 / 2)$ and the theorem is proved.

It is natural to ask whether the functions of class $\mathscr{S}$ are characterized completely by the properties I have just shown for their spiral limit value function. In other words, suppose a spiral limit value function is given having the properties stated in the preceding theorem (2.9). Can a function of class $\mathscr{S}$ then be constructed that has this given function as its associated spiral limit value function? Not only is the answer to this question in the affirmative, but the desired function can even be constructed so as to be holomorphic. It is this construction that is the concern of the remainder of this section. The following two theorems are essential to constructions I will make later on. I will list them here and refer to them as needed.

(2. 10) Theorem (Mergelyan). If $E$ is a closed bounded set not separating the plane, and if $f(z)$ is continuous on $E$ and analytic in the interior points of $E$, then $f(z)$ can be uniformly approximated on $E$ as closely as desired by a polynomial in $z$. (8, p. 367)

(2. 11) THEOREM (Walsh). Let the function $f(z)$ be given on the closed limited (i.e., bounded) point set $C$, and let distinct points $z_{1}, z_{2}, \ldots \ldots, z_{\nu}$ be given on $C$. If the function $f(z)$ can be uniformly approximated on $C$ as closely as desired by a polynomial in $z$, then the function can be uniformly approximated on $C$ as closely as desired by a polynomial $p(z)$ satisfying the auxiliary conditions

$$
p\left(z_{k}\right)=f\left(z_{k}\right) \quad k=1,2, \ldots \ldots, \nu .
$$

(8, p. 310).

(2. 12) Definition. A sequence of distinct Jordan curves $\left\{J_{n}\right\}_{1}^{\infty}$, shall be called increasing if $J_{n}$ lies in the interior of $J_{n+1}$ for $n=1,2, \ldots \ldots$. I shall say that such a sequence converges to the circle $C(k)$ if the sequence is contained in $D(k)$ and for any $\varepsilon>0$ there exists $n_{0}=n_{0}(\varepsilon)$ such that for every $n>n_{0}, J_{n}$ lies in the region $k-\varepsilon<|z|<k$. If $\left\{J_{n}\right\}_{1}^{\infty}$ is an increasing sequence I shall define $D_{n}$ to be the closed region bounded by and including $J_{n}$ for $n=1,2, \ldots \ldots$ and $D_{0}=\phi$. Then I define $A_{n}=\overline{D_{n+1}-D_{n}}$ for $n=0,1,2, \ldots \ldots$

(2. 13) Theorem. Let $\left\{G_{n}\right\}_{1}^{\infty}$ be a sequence of open, disjoint, connected sets in $D(1)$ such that $G_{n}$ contains the closed (i.e., closed in $\left.D(1)\right)$ set $M_{n}$ for each $n=1,2, \ldots \ldots$ Let $f_{n}(z)$ be defined and holomorphic for $z \in G_{n}$ for each $n=1,2, \ldots \ldots$ Given $\varepsilon \cdot>0$, the following conditions are sufficient for the existence of a function $F(z)$ holomorphic in $D(1)$ and satisfying 


$$
\left|F(z)-f_{n}(z)\right|<\varepsilon \quad \text { for } z \in M_{n}, \quad n=1,2, \ldots \ldots
$$

1. There exists a sequence of closed sets $\left\{B_{n}\right\}_{1}^{\infty}$ having no cluster point* in $D(1)$ such that for each $n, M_{n}$ is contained in the interior of $B_{n}$ and $B_{n}$ is contained in $G_{n}$.

2. There exists an increasing sequence of distinct Jordan curves $\left\{J_{n}\right\}_{1}^{\infty}$ in $D(1)$ converging to $C(1)$ such that if $b_{n}$ is the boundary of $B_{n}$,

a) $J_{m_{0}} \cap b_{n}$ is a finite point set for each $m$ and $n$.

b) $A_{m} \cap b_{n}$ is a finite number of arcs, each with a finite length, for each $m$ and $n$.

c) $D_{n} \cup\left(A_{n} \cap\left\{\bigcup_{k=1}^{\infty} b_{k}\right\}\right)$ does not separate the plane for $n=0,1, \ldots \ldots$

Proof. Since $M_{n}$ is contained in the interior of $B_{n}$ for each $n, b_{n} \cap M_{n}=\phi$ for each $n$. For $k \neq n, B_{k} \cap B_{n}=\phi$ since $G_{k} \cap G_{n}=\phi$ and so $b_{k} \cap M_{n}=\phi$. Therefore the sets $\bigcup_{k=1}^{\infty} b_{k}$ and $\bigcup_{k=1}^{\infty} M_{k}$ have no point in common. Moreover, both of these sets are closed in $D(1)$ since each term is closed and neither sequence has a cluster point in $D(1)$.

Let $p_{n}=\left(\bigcup_{k=1}^{\infty} b_{k}\right) \cap A_{n}$ for $n=0,1, \ldots \ldots . \quad p_{n}$ is closed and so has a positive distance $S_{n}$ from $\bigcup_{k=1}^{\infty} M_{k}, n=0,1,2, \ldots \ldots$. Since $\left\{B_{n}\right\}$ has no cluster point in $D(1)$, at most a finite number of terms of this sequence can have a non-empty intersection with $A_{n}$ for $n=0,1,2, \ldots \ldots$. Therefore, since $A_{n} \cap b_{m}$ is a finite number of arcs each with a finite length for each $m, p_{n}$ has a finite length which I shall designate by $l_{n}$ for $n=0,1,2$,

For each $S_{n}>0$ and $l_{n} \geq 0$ I define a positive number $\varepsilon_{n}$ so small that

$$
\sum_{n=0}^{\infty} \frac{\varepsilon_{n} \cdot l_{n}}{S_{n}}<2 \pi \varepsilon
$$

and

$$
\sum_{n=0}^{\infty} \varepsilon_{n} \cdot l_{n}<1
$$

Since $b_{n} \subset G_{n}$ for each $n, f_{n}(z)$ is continuous on $b_{n}$ for each $n$. Moreover, since the terms of $\left\{b_{n}\right\}$ are pairwise disjoint and the sequence has no cluster point in $D(1)$, a continuous function $f(z)$ can be defined on $\bigcup_{k=1}^{\infty} b_{k}$ by the correspondence $f(z)=f_{n}(z)$ for $z$ on $b_{n} \quad n=1,2, \ldots \ldots$.

* A point is a cluster point of a sequence $\left\{B_{n}\right\}$ if every one of its neighborhoods contains a point from an infinite number of terms of the sequence. 
Let $\varepsilon(r)$ be a positive continuous function for $0 \leq r<1$ such that $\lim _{r \rightarrow 1} \varepsilon(r)=0$. I shall now construct a function $F_{1}(z)$ holomorphic in $D(1)$ such that for $z \in \bigcup_{k=1}^{\infty} b_{k}$ $\left|F_{1}(z)-f(z)\right|<\varepsilon(|z|)$.

To this end let $\varepsilon_{0}^{\prime}, \varepsilon_{1}^{\prime}, \ldots \ldots$ be positive numbers such that $\varepsilon_{n}^{\prime}>\varepsilon_{n+1}^{\prime}$ for all $n$ and $\varepsilon_{n}^{\prime}<\varepsilon(r)$ for $r \leq \max _{z \in J_{n+1}}|z|$ for $n=0,1,2, \ldots \ldots$. Let $\eta_{n}=\varepsilon_{n}^{\prime}-\varepsilon_{n+1}^{\prime}$ so that $\eta_{0}, \eta_{1}$, are positive numbers and $\sum_{\nu=n}^{\infty} \eta_{\nu}=\varepsilon_{n}^{\prime} \quad n=0,1,2, \ldots \ldots$.

As has already been pointed out, at most a finite number of terms in the sequence $\left\{B_{n}\right\}$ have a non-empty intersection with $J_{\nu+1}$ for each $\nu$. Since $b_{k} \cap J_{\nu+1}$ is a finite point set for each $k,\left(\bigcup_{k=1}^{\infty} b_{k}\right) \cap J_{\nu+1}$ contains at most a finite number of points which I shall designate as $z_{1}^{(\nu)}, z_{2}^{(\nu)}, \ldots \ldots, z_{n(\nu)}^{(\nu)}$. By Condition $2 c$ in the hypothesis, for $\nu=0,1,2, \ldots \ldots, D_{\nu} \cup p_{\nu}$ is a closed bounded set that does not separate the plane.

Now, by induction, for $\nu=0, D_{0} \cup p_{0}$ satisfies the condition of the theorems in Sections (2.10) and (2. 11) and so I may conclude that there is a polynomial $\pi_{0}(z)$ such that $\left|f(z)-\pi_{0}(z)\right|<\eta_{0}$ for $z \in D_{0} \cup p_{0}$ and $f\left(z_{k}^{0}\right)=\pi\left(z_{k}^{0}\right)$ for $k=1,2, \ldots \ldots$, $n(0)$.

Assume that $\nu>0$ and polynomials $\pi_{0}(z), \ldots \ldots, \pi_{\nu-1}(z)$ have been defined so that $\pi_{\nu-1}\left(z_{k}^{\nu-1}\right)=f\left(z_{k}^{\nu-1}\right)-\pi_{0}\left(z_{k}^{\nu-1}\right)-\cdots \cdots-\pi_{\nu-2}\left(z_{k}^{\nu-1}\right)$ for $k_{k}=1,2, \ldots \ldots, n(\nu-1)$. Then the theorems in Sections (2.10) and (2.11) assert the existence of a polynomial $\pi_{\nu}(z)$ so that $\left|\pi_{\nu}(z)\right|<\eta_{\nu}$ for $z \in D_{\nu},\left|f(z)-\left[\pi_{0}(z)+\pi_{1}(z)+\cdots \cdots+\pi_{\nu}(z)\right]\right|<\eta_{\nu}$ for $z \in p_{\nu}$, and $\pi_{\nu}\left(z_{k}^{\nu}\right)=f\left(z_{k}^{\nu}\right)-\sum_{n=0}^{\nu-1} \pi_{n}\left(z_{k}^{\nu}\right), k=1, \ldots \ldots, n(\nu)$. Now define $F_{1}(z)=\sum_{\nu=0}^{\infty} \pi_{\nu}(z)$.

For $z \in D_{k}$, since $D_{k} \subset D_{k+n}$ for all $n \geq 1,\left|\pi_{\nu}(z)\right|<\eta_{\nu}$ for $\nu=k, k+1, \ldots \ldots$. Also there are constants $\mathscr{M}_{\nu}>0$ such that $\left|\pi_{\nu}(z)\right|<\mathscr{M}_{\nu}$ for $\nu=1,2, \ldots \ldots, k-1$ because $\pi_{\nu}(z)$ is a polynomial and $D_{k}$ is compact. Since $\sum_{\nu=k}^{\infty} \eta_{\nu}$ converges, by the Weierstrass M-test, $\sum_{\nu=0}^{\infty} \pi_{\nu}(z)$ is uniformly convergent on $D_{k}$. But every compact subset of $D(1)$ is contained in $D_{k}$ for some $k$. Therefore $F_{1}(z)=\sum_{\nu=0}^{\infty} \pi_{\nu}(z)$ converges uniformly on every compact subset of $D(1)$ and so represents a holomorphic function in $D(1)$.

Moreover, for $z \in p_{k},\left|f(z)-F_{1}(z)\right|=\left|f(z)-\sum_{\nu=0}^{\infty} \pi_{\nu}(z)\right| \leq\left|f(z)-\sum_{\nu=0}^{k} \pi_{\nu}(z)\right|+\sum_{\nu=k+1}^{\infty}\left|\pi_{\nu}(z)\right|$ $<\eta_{k}+\sum_{\nu=k+1}^{\infty} \eta_{\nu}$ since $p_{k} \subset D_{k+n}$ for $n \geq 1$. But $\sum_{\nu=k}^{\infty} \eta_{\nu}<\varepsilon_{k}^{\prime}<\varepsilon(r)$ for $r \leq \max _{z \in J_{k+1}}|z|$ and 
so in particular for all $z \in p_{k}$. Since $k$ is arbitrary, for any $z \in \bigcup_{k=0}^{\infty} p_{k}=\bigcup_{n=1}^{\infty} b_{n}$, $\left|f(z)-F_{1}(z)\right|<\varepsilon(|z|)$ and the construction is finished.

Using this function $F_{1}(z)$ and the construction employed by Roth (6, pp. $107-$ $109)$, the desired function $F(z)$ results.

(2. 14) Corollary. If each of the regions $G_{n}$ in (2.13) can be mapped conformally onto a subset of $\left|z^{\prime}\right|<1$ by a function that has a continuous extension onto the boundary of $G_{n}$ and also maps $\bar{G}_{n} \cap C(1)$ onto the single point $z^{\prime}=1$, then there exists $a$ function $F(z)$ holomorphic in $D(1)$ such that $\left|F(z)-f_{n}(z)\right|<\varepsilon$ for $z \in M_{n} \quad(n=1,2$, ......) and also $\left[F(z)-f_{n}(z)\right]$ tends uniformly to 0 as $|z|$ tends to $1\left(z \in M_{n} ; n=1,2, \ldots \ldots\right)$.

Proof. If the given mapping is possible, $G_{n}$ can be mapped conformally onto $H_{n}$, a subset of the following region $H$ in the $\zeta$-plane, in such a way that $\bar{G}_{n} \cap C(1)$, which I shall call $E_{n}$, corresponds to the point $\zeta=-\infty$.

$$
H: \quad R(\zeta)<-1 \quad \text { and } \quad|I(\zeta)|<1 .
$$

Let $\zeta=\zeta_{n}(z)$ designate this mapping and let $z=z_{n}(\zeta)$ be the inverse of $\zeta=\zeta_{n}(z)$.

For $N$ sufficiently large, the line $\zeta=-N+i v,-1<v<+1$, cuts the region $H_{n}$ and divides it into two subsets $H_{n}^{1}$ and $H_{n}^{2}$ where $H_{n}^{2}$ represents the unbounded subset. Let $G_{n}^{1}$ and $G_{n}^{2}$ be the images of $H_{n}^{1}$ and $H_{n}^{2}$ respectively under $z=z_{n}(\zeta)$. If $\left\{z_{n}\right\}$ is a sequence of points in $G_{n}^{1},\left|\zeta\left(z_{n}\right)\right|<N+1$ and so no sequence in $G_{n}^{1}$ can converge to a point of $E_{n}$ and so not to a point of $C(1)$. Therefore there is a positive distance $d(N)$ between $\bar{G}_{n}^{1}$ and $C(1)$. If $z \in G_{n}$ and $|z|>1-d(N)$ then $z$ must be in $G_{n}^{2}$ and thus $R\left(\zeta_{n}(z)\right)<-N$ (i.e., the real part of $\left.\zeta_{n}(z)<-N\right)$.

Now I have $\zeta_{n}(z)$ holomorphic in $G_{n}$ for each $n=1,2, \ldots \ldots$; and for $z \in M_{n} \subset G_{n}$ and $|z|>1-d(N)$ this implies that $R\left(\zeta_{n}(z)\right)<-N$. So, $R\left(\zeta_{n}(z)\right) \rightarrow-\infty$ uniformly as $|z| \rightarrow 1$ in $M_{n}(n=1,2, \ldots \ldots)$.

Therefore, by (2. 13), there exists a function $F^{*}(z)$ holomorphic in $D(1)$ such that $\left|F^{*}(z)-\zeta_{n}(z)\right|<\varepsilon$ for $z \in M_{n}(n=1,2, \ldots \ldots)$. Since $R\left(\zeta_{n}(z)\right) \rightarrow-\infty$ uniformly as $|z| \rightarrow 1$ in $M_{n}, n=1,2, \ldots \ldots, R\left(F^{*}(z)\right) \rightarrow-\infty$ uniformly as $|z| \rightarrow 1$ in $M_{n} \quad n=1$, $2, \ldots . .$. .

Since $F^{*}(z)$ is holomorphic in $D(1), w(z)=e^{F^{*}(z)}$ is holomorphic in $D(1)$ and $w(z) \neq 0$ for $z \in D(1)$. Furthermore, $|w(z)|=e^{R\left(F^{*}(z)\right)} \rightarrow 0$ uniformly as $|z| \rightarrow 1$ for $z \in M_{n}, n=1,2, \ldots \ldots$.

Since $f_{n}(z)$ is holomorphic in $G_{n}$ for each $n$ and $w(z) \neq 0$ for any $z$ in $G_{n}$ for each $n, f_{n}(z) / w(z)$ represents a holomorphic function in $G_{n}$ for each $n=1,2, \ldots \ldots$. 
By (2. 13), there exists a function $F^{* *(z)}$ holomorphic in $D(1)$ such that $\left|F^{* *}(z)-f_{n}(z) / w(z)\right|<\varepsilon$ for $z \in M_{n} n=1,2, \ldots \ldots$. Let $F(z)=F^{* *}(z) \cdot w(z)$. Then $F(z)$ is holomorphic for $z \in D(1)$ and $\left|F(z)-f_{n}(z)\right|<\varepsilon|w(z)|$ for $z \in M_{n} n=1,2$, ...... Since $|w(z)|$ tends uniformly to 0 as $|z|$ tends to 1 for $z \in M_{n} \quad(n=1,2$, .....), the theorem is proved.

I have now developed enough technique to proceed with the details of the answer presented on page 260 .

(2.15) Theorem. Let $\Delta$ be a non-empty closed subset of $C(1 / 2)$ which is nowhere dense on $C(1 / 2)$. Let $f(\varphi)$ (which may assume the value $\infty$ ) be defined for $0 \leq \varphi<2 \pi$ such that when $f(\varphi)$ is associated by $g$ with each point $1 / 2 \exp [i(\varphi+\tan \pi / 4)]$ of $C(1 / 2) 0 \leq \varphi<2 \pi$ the correspondence is a function of Baire class 0 or 1 and $g$ is constant at least on each circular arc disjoint from the set $\Delta$. Then there exists a function $F(z)$ holomorphic in $D(1)$ such that:

1) $\lim _{r \rightarrow 1} F\left(r \exp \left[i\left(\varphi+\tan \frac{\pi r}{2}\right)\right]\right)=f(\varphi) \quad 0 \leq \varphi<2 \pi$.

2) If $W$ is a closed spiral wedge which contains no point of $\Delta$ and $f(\varphi)$ is the corresponding constant spiral limit value, $F(z)$ tends uniformly to $f(\varphi)$ as $|z|$ tends to 1 , $z \in W$.

3) Every Study spiral cutting $C(1 / 2)$ at a point of $\Delta$ is a Julia spiral.

Proof.* Since $f(\varphi)$ is defined on $C(1 / 2)$ and is of Baire class 0 or 1 , there exists a sequence of functions $f_{1}(\varphi), f_{2}(\varphi), \ldots \ldots$ which are continuous on $C(1 / 2)$ and converge to $f(\varphi)$. Let $f(\varphi)=r_{0}(\varphi) \exp \left[i \theta_{0}(\varphi)\right]$ where $r_{0}(\varphi)$ and $\theta_{0}(\varphi)$ are continuous functions of $\varphi$. Now define $f_{n}(\varphi)=r_{n}(\varphi) \exp \left[i \theta_{n}(\varphi)\right]$ where $\theta_{n}(\varphi)$ $=\theta_{0}(\varphi)$ if $r_{n}(\varphi)=0$. Since $\lim _{n \rightarrow \infty} f_{n}(\varphi)=f(\varphi)$, there is a proper determination of $\theta_{n}(\varphi)$ for each $n$ and each $\varphi$ such that $\lim _{n \rightarrow \infty} \theta_{n}(\varphi)=\theta_{0}(\varphi)$ and $\lim _{n \rightarrow \infty} r_{n}(\varphi)=r_{0}(\varphi)$.

Let $d_{r}^{(n)}=\frac{\frac{n}{n+1}-r}{\frac{n}{n+1}-\frac{n-1}{n}}$ for $n=1,2, \ldots \ldots$ and $\frac{n-1}{n}<r \leq \frac{n}{n+1}$. Then $0 \leq d_{r}^{(n)}<1, n=1,2, \ldots \ldots \ldots$. Let $h(0)=0=f_{0}(\varphi)$. Then for $\frac{n-1}{n}<r \leq \frac{n}{n+1}$ set $h(r \exp [i(\varphi+\tan \pi r / 2)])=\left\{r_{n}(\varphi)+d_{r}^{(n)}\left(r_{n-1}(\varphi)-r_{n}(\varphi)\right)\right\} \exp \left[i\left(\theta_{n}(\varphi)+d_{r}^{(n)}\left\{\theta_{n-1}(\varphi)\right.\right.\right.$ $\left.\left.\left.-\theta_{n}(\varphi)\right\}\right)\right]$. Then $h(z)$ is continuous in $D(1)$ and it can be easily verified that $\lim _{r \rightarrow 1} h(r \exp [i(\varphi+\tan \pi r / 2)])=f(\varphi)$.

* The argument used by Roth $(6$, p. 117$)$ to construct a function $h(z)$ continuous in the entire plane does not hold if $f(\varphi)=\infty$ for some $\varphi$. A slight modification of the construction of the function $h(z)$ used in the first part of this proof can be used to correct this error. 
Let $\Gamma$ be the set of all Study spirals that intersect the set $\Delta$ on $C(1 / 2)$. Then $\Gamma$ is closed and nowhere dense in $D(1)$. It follows from a theorem of Bagemihl and Seidel (1, p. 190, Corollary 1) that there exists a function $G_{\Gamma}(z)$ holomorphic in $D(1)$ such that if $1 / 2 \exp [i(\varphi+\tan \pi / 4)]$ is in $\Delta$ then $\lim _{r \rightarrow 1} G_{\Gamma}(r \exp [i(\varphi$ $+\tan \pi r / 2)])=\lim _{r \rightarrow 1} h(r \exp [i(\varphi+\tan \pi r / 2)])=f(\varphi)$.

The complementary set $G=C(1 / 2)-\Delta$ is an open subset of $C(1 / 2)$ and so consists of countably many pairwise disjoint open $\operatorname{arcs} g_{0}, g_{1}, g_{2}, \ldots \ldots$. Let $W_{n}$ be the spiral wedge for which $W_{n} \cap C(1 / 2)=g_{n} \quad n=0,1,2, \ldots \ldots$. Let $W_{n}=W\left(\alpha_{n}, \beta_{n}\right)$ where $S_{\alpha_{n}}$ and $S_{\beta_{n}}$ are the Study spirals that bound $W_{n}, \alpha_{n}<\beta_{n}$. Let $C_{n}$ (finite or infinite) be the constant value of $f(\varphi)$ associated with the arc $g_{n}$. Let $r_{k}^{(n)}=\frac{n}{n+1}+\frac{1}{k}\left(\frac{n+1}{n+2}-\frac{n}{n+1}\right) \quad(k=3,4, \ldots \ldots, 10 ; n=0,1,2, \ldots \ldots)$. Define $\Sigma_{n}(k)=\left\{z \mid z=r \exp \left[i\left(\alpha_{n}+\tan \frac{\pi r}{2}+\sigma_{k}^{(n)}(r)\right)\right], 1>r \geq r_{k}^{(n)}\right\} \cup\left\{z \mid z=r \exp \left[i\left(\beta_{n}\right.\right.\right.$ $\left.\left.\left.+\tan \frac{\pi r}{2}-\sigma_{k}^{(n)}(r)\right)\right], 1>r \geq r_{k}^{(n)}\right\} \cup\left\{z=r_{k}^{(n)} \exp i \theta \mid \alpha_{n}+\tan \frac{\pi r_{k}^{(n)}}{2}+\sigma_{k}^{(n)}\left(r_{k}^{(n)}\right) \leq \theta\right.$ $\left.\leq \beta_{n}+\tan \frac{\pi r_{k}^{(n)}}{2}-\sigma_{k}^{(n)}\left(r_{k}^{(n)}\right)\right\}$ where $\sigma_{k}^{(n)}(r)=(1-r) \frac{\left(\beta_{n}-\alpha_{n}\right)}{k}(k=3,4, \ldots \ldots, 10 ; n=0$, $1,2, \ldots \ldots)$. Notice that $\lim _{r \rightarrow 1} \sigma_{k}^{(n)}(r)=0$ for all $k$ and $n$. Thus $\Sigma_{n}(k)$ "opens up" in $W_{n}$ as $r \rightarrow 1$ and its "ends" approach $S_{\alpha_{n}}$ and $S_{\beta_{n}}$ asymptotically from within $W_{n}$ as $r \rightarrow 1$. Let $\lambda_{n}=\Sigma_{n}(7)$. Let $B\left(\lambda_{n}\right)$ be the closed region bounded by $\Sigma_{n}(6)$ and $\Sigma_{n}(8)$ and containing $\lambda_{n}$. Let $G\left(\lambda_{n}\right)$ be the open region bounded by $\Sigma_{n}(5)$ and $\Sigma_{n}(9)$. Then $B\left(\lambda_{n}\right)$ is closed and contains $\lambda_{n}$ in its interior and $B\left(\lambda_{n}\right)$ is contained in $G\left(\lambda_{n}\right)(n=0,1,2, \ldots \ldots)$.

Let $\tau_{n}$ be the closed region entirely contained in $W_{n}$ which is bounded by $\Sigma_{n}(3)$. Define $B\left(\tau_{n}\right)$ to be the closed region bounded by $\Sigma_{n}(4)$ and containing $\tau_{n}$ in its interior. Let $G\left(\tau_{n}\right)$ be the open region bounded by $\Sigma_{n}(5)$ and containing $B\left(\tau_{n}\right)$.

Lastly, let $B(\Gamma)$ be the closed region bounded by $\bigcup_{n=0}^{\infty} \Sigma_{n}(10)$ and containing $\Gamma$ in its interior. Let $G(\Gamma)$ be the open region bounded by $\bigcup_{n=0}^{\infty} \Sigma_{n}(9)$ and containing $B(\Gamma)$ in its interior.

If I define $J_{n}=\left\{z|| z \mid=\frac{n}{n+1}\right\}(n=1,2, \ldots \ldots)$ then $\left\{J_{n}\right\}$ is an increasing sequence of distinct Jordan curves converging to $C(1)$. Consider the open regions $G(\Gamma), G\left(\lambda_{0}\right), G\left(\tau_{0}\right), G\left(\lambda_{1}\right), G\left(\tau_{1}\right), \ldots \ldots$ and, for the sake of simple notation, 


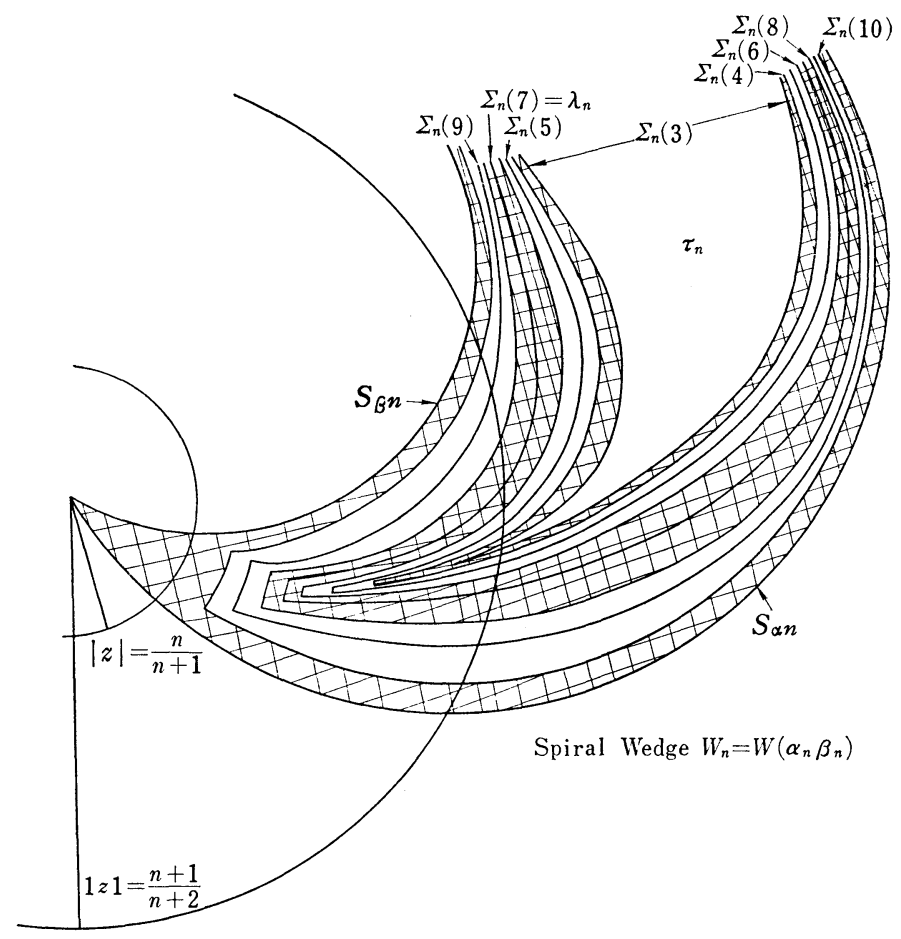

rename these regions $G_{1}, G_{2}, \ldots \ldots$. Each region contains a certain closed set (e.g., $G(\Gamma) \supset \Gamma, G\left(\lambda_{i}\right) \supset \lambda_{i}$, and $\left.G\left(\tau_{i}\right) \supset \tau_{i}\right)$ and I shall correspondingly label these $M_{1}, M_{2}, \ldots \ldots$ The closed set contained in $G_{i}$ and containing $M_{i}$ in its interior I shall call $B_{i}$ (e.g., $G_{i}=G\left(\tau_{i}\right), B_{i}=B\left(\tau_{i}\right)$ and $M_{i}=\tau_{i}$ ). It is immediate at this point that the sequences $\left\{G_{n}\right\}_{1}^{\infty},\left\{B_{n}\right\}_{1}^{\infty},\left\{M_{n}\right\}_{1}^{\infty}$ and $\left\{J_{n}\right\}_{1}^{\infty}$ have been constructed to satisfy the conditions stated in (2. 13).

Now consider $\Sigma_{0}(9)$. It is disjoint from $G_{n}$ for each $n=0,1, \ldots \ldots$. Let $\sigma$ represent that part of $\Sigma_{0}(9)$ defined by $\left\{z \mid z=r \exp i\left(\beta_{0}+\tan \frac{\pi r}{2}-\sigma_{9}^{(0)}(r)\right)\right] 1>r$ $\left.\geq r_{9}^{(0)}\right\}$. This $\sigma$ is a spiral in $D(1)$ in the $z$-plane. Let $G=D(1)-\sigma$. Let $D^{\prime}(1)$ represent the set $|\zeta|<1$. The initial point of $\sigma$ is the impression of one prime end of $G$, while every other point of $\sigma$ is the impression of two prime- ends of $G$. Since $\sigma$ converges to $C(1), C(1)$ is the impression of a single prime end $P$ of $G$. Using Carathéodory's classical results (3), there exists a one-to-one con- 
formal mapping from $G$ onto the unit disk $D^{\prime}(1)$ so that the initial point of $\sigma$ and the prime end $P$ correspond, respectively, to the points $-1,1$ while the other points of $\sigma$ are mapped onto $C^{\prime}(1)-\{1,-1\}$. Let $\zeta=\zeta_{\sigma}(z)$ be the mapping from $G$ to $D^{\prime}(1)$ and $z=z_{\sigma}(\zeta)$ the inverse of this mapping.

Each of the regions $G_{n}$ has the property that $\bar{G}_{n} \cap C(1)=C(1)$. Furthermore $\zeta_{\sigma}(z)$ restricted to $G_{n}$ has a continuous extension onto $\bar{G}_{n}$ which maps $C(1)$ onto the single point $\zeta=1$ for each $n$. Therefore the continuous extension of $\zeta_{\sigma}(z)$ restricted to $\bar{G}_{n}$ satisfies the condition stated in (2. 14) for each $n=0,1, \ldots \ldots$.

It remains only for me to define a function $f_{n}(z)$ holomorphic in $G_{n}(n=0,1$, ......) in order for me to be able to use the conclusions of (2.13) and (2.14). To do this I must consider the regions $G_{1}, G_{2}, \ldots \ldots$ as formerly represented: $G(\Gamma), G\left(\lambda_{0}\right), G\left(\tau_{0}\right), \ldots \ldots$.

Let $G_{\Gamma}(z)$ be the holomorphic function defined on the region $G(\Gamma)$. If the constant $C_{n}$ associated with $W_{n}$ is finite, let the function $f^{*}(z) \equiv C_{n}$ be the holomorphic function defined on $G\left(\tau_{n}\right)$. Then define $f^{* *}(z)=1 / \zeta_{\sigma}(z)-1$ for $z \in G\left(\lambda_{n}\right)$. This function is then holomorphic on $G\left(\lambda_{n}\right)$ and tends uniformly to $\infty$ as $|z|$ tends to 1 in $G\left(\lambda_{n}\right)$.

On the other hand, if $C_{n}=\infty$ for $W_{n}$, define $f^{*}(z)=1 / \zeta_{\sigma}(z)-1$ for $z \in G\left(\tau_{n}\right)$. This function is then holomorphic in $G\left(\tau_{n}\right)$ and tends to $\infty$ uniformly as $|z|$ tends to $1, z \in G\left(\tau_{n}\right)$. Then define $f^{* *}(z) \equiv 0$ for $z \in G\left(\lambda_{n}\right)$.

Thus, reverting back to the simpler notation, with each region $G_{n}$ I have associated a function $f_{n}(z)$ holomorphic there. Given $\varepsilon>0,(2.14)$ allows me to conclude the existence of a function $F(z)$ holomorphic in $D(1)$ such that $\left|F(z)-f_{n}(z)\right|<\varepsilon$ for $z \in M_{n}(n=1,2,3, \ldots \ldots)$ and also $\left[F(z)-f_{n}(z)\right]$ tends uniformly to zero as $|z|$ tends to $1, z \in M_{n}(n=1,2, \ldots \ldots)$.

Now consider the Study spiral $S_{\varphi}$. For some $n, S_{\varphi}$ is eventually in $M_{n}$. Hence there is an $R, 0<R<1$ such that for $|z|=r>R$ and $z \in S_{\varphi}, z \in M_{n}$. If $f(\varphi)$ is finite, $f_{n}(z)=f(\varphi)$ for $z \in S_{\varphi}$ and $|z|>R$ and so $\lim _{r \rightarrow 1} \mid F\left(r \exp \left[i\left(\varphi+\tan \frac{\pi r}{2}\right)\right]\right)$ $-f(\varphi)\left|\leq \lim _{r \rightarrow 1}\right| F\left(r \exp \left[i\left(\varphi+\tan \frac{\pi r}{2}\right)\right]\right)-f_{n}\left(r \exp \left[i\left(\varphi+\tan \frac{\pi r}{2}\right)\right]\right) \mid$ $+\lim _{r \rightarrow 1}\left|f_{n}\left(r \exp \left[i\left(\varphi+\tan \frac{\pi r}{2}\right)\right]\right)-f(\varphi)\right|=0$. Thus, for $f(\varphi)$ finite, $\lim _{\substack{|z| \rightarrow 1 \\ z \in S_{\varphi}}} F(z)=f(\varphi)$. If, on the other hand, $f(\varphi)=\infty$, for $z \in S_{\varphi}$ and $|z|>R$ it is true that $\left|F(z)-f_{n}(z)\right|$ $<\varepsilon$. This implies that $\left|f_{n}(z)\right|-\varepsilon<|F(z)|$ and since $\lim _{\substack{|z| \rightarrow 1 \\ z \in S_{\varphi}}}\left|f_{n}(z)\right|=\infty$ it follows that $\lim _{\substack{|z| \rightarrow 1 \\ z \in S_{\varphi}}}|F(z)|=\infty$ or $\lim _{\substack{|z| \rightarrow \mid \\ z \in S_{\varphi}}} F(z)=f(\varphi)$. 
Now suppose $W$ is a closed spiral wedge which contains no point of $\Delta$. Then $W \cap C(1 / 2)$ is contained entirely in $g_{m}$ for some $m$. It follows then that $W$ is eventually contained in $M_{n}$ for some $n$. That is, there exists $0<R<1$ such that for $z \in W$ and $|z|>R, z \in M_{n}$.

If the corresponding constant spiral limit value $C_{m}$ is finite, $f_{n}(z)=C_{m}$ for all $z \in M_{n}$, so $\lim _{\substack{|z| \rightarrow 1 \\ z \in W}}\left|F(z)-C_{m}\right|=\lim _{\substack{|z| \rightarrow 1 \\ z \in M_{n}}}\left|F(z)-f_{n}(z)\right|$ and since $\left[F(z)-f_{n}(z)\right]$ tends to zero uniformly as $|z|$ tends to 1 for $z \in M_{n}$, it follows that $F(z)$ tends uniformly to the corresponding constant limit value as $|z| \rightarrow 1$ for $z \in W$.

If $C_{m}=\infty, f_{n}(z)$ tends to $\infty$ uniformly as $|z| \rightarrow 1$ and $z$ remains in $M_{n}$. If $z \in W$, and $|z|>R, z$ must be in $M_{n}$. If $z \in W$ and $|z|>R$, it follows that $\left|F(z)-f_{n}(z)\right|<\varepsilon$. Then $\left|f_{n}(z)\right|-\varepsilon<|F(z)|$ and $|F(z)|$ must tend to $\infty$ uniformly as $|z| \rightarrow 1$ for $z \in W$. Thus, in this case also, $F(z)$ tends uniformly to the corresponding constant limit value as $|z|$ tends to 1 for $z \in W$.

Lastly consider any spiral wedge $W^{*}$ which contains a point of $\Delta$. Then $W^{*}$ contains the Study spiral through that point and so cannot be a wedge of convergence. Since the $\operatorname{arcs} g_{1}, g_{2}, \ldots \ldots$ are everywhere dense on $C(1 / 2)$ and since $W^{*} \cap C(1 / 2)$ is not contained entirely in any one of them, $W^{*} \cap C(1 / 2)$ contains at least a part of $g_{n}$ for some $n$. Thus $W^{*}$ contains at least a terminal part of $\lambda_{n}$. Then $W^{*}$ contains spirals along which $|F(z)|$ is both bounded and unbounded and so $|F(z)|$ is neither bounded or uniformly unbounded in $W^{*}$ as $|z| \rightarrow 1$ and thus $W^{*}$ cannot be a wedge of convergence. Since $I$ have previously shown that the set of Julia spirals is identical with the set of Study spirals that are contained in no wedge of convergence, it follows that each Study spiral cutting $C(1 / 2)$ at a point of $\Delta$ is a Julia spiral and the theorem is complete.

\section{An extension to a larger class}

Harold Bohr has considered (2) the class of entire functions that are bounded on each half line emanating from the origin. This class, of course, includes much of the class of functions considered by Roth and it is natural to ask whether analogous results can be obtained for functions holomorphic in $D(1)$ where the class of spirals in the disk plays the role of half lines in the plane. For my purposes I again consider the class of Study spirals as rather natural analogues in the disk of the half lines in the plane, but of course make no claim as to their exclusiveness.

(3. 1) Definition. $M^{*}$ shall denote the class of functions $F(z)$ holomor- 
phic in $D(1)$ and bounded on each Study spiral.

It follows from this that if $F(z)$ is in $M^{*}$ for each $\theta(0 \leqslant \theta<2 \pi)$ there is a real positive number $L(\theta)$ such that for all $z \in S_{\theta},|F(z)|<L(\theta)$.

I shäll use $i$ to denote an open connected subset of $C(1 / 2)$ and I shall distinguish between three distinct types of intervals as follows:

1) The entire circle, therefore an interval without frontier points, which I shall designate as I.

2) An interval with exactly one frontier point $A=1 / 2 \exp \left[i\left(\alpha+\tan \frac{\pi}{4}\right)\right]$, therefore the entire circle except this point, which I shall designate by $I_{A}$.

3) An interval with two frontier points $A=1 / 2 \exp \left[i\left(\alpha+\tan \frac{\pi}{4}\right)\right]$ and $B=$ $1 / 2 \exp \left[i\left(\beta+\tan \frac{\pi}{4}\right)\right]$. I shall designate this interval by $I_{A B}$ where the order of the two frontier points is so chosen that as $z$ moves from $A$ to $B$ on $I_{A B}$ it travels in the counterclockwise direction.

(3. 2) Definition. I shall say that $F(z) \in M^{*}$ is bounded on an interval $i$ if $F(z)$ is uniformly bounded on all Study spirals which pass through a point of $i$. That is, there must exist a constant $B(i)$ such that for all $\theta$ for which $S_{\theta}$ cuts $i, L(\theta)<B(i)$.

(3.3) Definition. Concerning two intervals $i_{1}$ and $i_{2}$, I shall say that $i_{1}$ is a subinterval of $i_{2}$ iff not only all points of $i_{1}$ are contained in $i_{2}$ but also all frontier points of $i_{1}$ are also in $i_{2}$.

(3. 4) Definition. An interval $i$ is called a complete interval of boundedness for a function $F(z) \in M^{*}$ iff 1) $F(z)$ is bounded in every subinterval of $i$ and 2) $F(z)$ is not bounded in any interval which contains a frontier point of $i$.

It follows that two complete intervals of boundedness for a given function are either disjoint or identical. Also, if $F(z)$ is bounded on an interval $i$, there is a unique interval $i *$ containing $i$ which is a complete interval of boundedness.

(3. 5) Theorem. If $F(z) \in M^{*}$ and $i$ is an arbitrarily small interval on $C(1 / 2)$, then $i$ contains a subinterval on which $F(z)$ is bounded. Consequently the complete intervals of boundedness are everywhere dense on $C(1 / 2)$.

Proof. Let $i$ be bounded by $1 / 2 \exp \left[i\left(\alpha_{0}+\tan \pi / 4\right)\right]$ and $1 / 2 \exp \left[i\left(\beta_{0}\right.\right.$ $+\tan \pi / 4)]$ where $\alpha_{0}<\beta_{0}<\alpha_{0}+2 \pi$ and let $F_{r}(\theta)=f(r \exp [i(\theta+\tan \pi r / 2)])$ for 
$\alpha_{0}<\theta<\beta_{0}$. If the theorem is not true, there exists $r_{1}$ and $\theta_{1}$ such that $\left|F_{r_{1}}\left(\theta_{1}\right)\right|>1$. Since $F_{r_{1}}$ is a continuous function of $\theta$, there exists an entire interval $\left(\alpha_{1}, \beta_{1}\right)$ containing $\theta_{1}$ where $\alpha_{0}<\alpha_{1}<\beta_{1}<\beta_{0}$ such that $\left|F_{r_{1}}(\theta)\right|>1$ for $\theta \in\left(\alpha_{1}, \beta_{1}\right)$.

If $\left(\alpha_{1}, \beta_{1}\right)$ is not an interval of boundedness, then there exists $r_{2}$ and $\theta_{2}$ such that $\left|F_{r_{2}}\left(\theta_{2}\right)\right|>2$. Again by continuity, there exists an entire interval $\left(\alpha_{2}, \beta_{2}\right)$ containing $\theta_{2}$ where $\alpha_{1}<\alpha_{2}<\beta_{2}<\beta_{1}$ such that $\left|F_{r_{2}}(\theta)\right|>2$ for $\theta \in\left(\alpha_{2}, \beta_{2}\right)$.

Continuing in this way, if no interval of boundedness is encountered, there exists $\theta_{0} \in \bigcap_{n=1}^{\infty}\left(\alpha_{n}, \beta_{n}\right)$ such that $\left|F_{r_{n}}\left(\theta_{0}\right)\right|>n$ for each positive integer $n$. But this denies the existence of $L\left(\theta_{0}\right)$ and so proves the theorem.

It is natural to ask, as in the previous section, whether a converse can be found for this theorem. The following is the affirmative answer.

(3.6) Theorem. Let $i_{1}, i_{2}, \ldots .$. be an arbitrary ( finite or countably infinite) set of intervals on $C(1 / 2)$ such that no two of these intervals possess a common point and the intervals are everywhere dense on $C(1 / 2)$. Then there exists a function of class $M^{*}$ whose complete intervals of boundedness are precisely the intervals $i_{1}, i_{2}, \ldots \ldots$.

Proof. If $I$ is the only interval that is given, any function constant in $D(1)$ will suffice. Otherwise the set $C(1 / 2)-\bigcup_{n=1}^{\infty} i_{n}$ is closed and not empty, and I shall call it $\Delta$. Now apply the theorem of Section 2.15 with $f(\varphi)=0$ for all $\varphi(0 \leq \varphi<2 \pi)$, and the conclusion follows immediately.

\section{REFERENCES}

[1] F. Bagemihl and W. Seidel, Some boundary properties of analytic functions. Math. Zeit. 61, 186-199 (1954).

[2] H. Bohr, UUber ganze transzendente Funktionen, die auf jeder durch den Nullpunkt gehenden Geraden beschränkt sind. Opusc. Math. A. Wiman Dedicata (1930).

[3] C. Carathéodory, Über die Begrenzung einfach zusammenhängender Gebiete. Math. Annalen 73, 323-370 (1913).

[4] O. Lehto and K.I. Virtanen, Boundary behaviour and normal meromorphic functions. Acta Math. 97, 47-65 (1957).

[5] K. Noshiro, Cluster Sets. Springer-Verlag, Berlin, 1960.

[6] A. Roth, Approximationseigenschaften und Strahlengrenzwerte meromorpher und ganzer Funktionen. Comment. Math. Helvet. 11, 77-125 (1938).

[7] E. Study, Vorlesungen über ausgewählte Gegenstände der Geometrie, Zweites Heft, Konforme Abbildung einfachzusammenhängender Bereiche. Leipzig and Berlin, 1913.

[8] J.L. Walsh, Interpolation and Approximation by Rational Functions in the Complex Plane. Amer. Math. Soc. Colloquium Publications. 20, Third Edition, 1960.

Wayne State University and The University of Wisconsin-Milwaukee 